\title{
Is MRI a viable alternative to $C T / C B C T$ to identify the course of the inferior alveolar nerve in relation to the roots of the third molars?
}

\author{
Florian Beck ${ }^{1}$ (D) $\cdot$ Stephanie Austermann ${ }^{1} \cdot$ Kristina Bertl $^{1,2} \cdot$ Christian Ulm $^{1} \cdot$ Stefan Lettner $^{3} \cdot$ Andrea Toelly $^{4}$. \\ André Gahleitner ${ }^{4}$
}

Received: 27 August 2020 / Accepted: 25 November 2020 / Published online: 7 December 2020

(C) The Author(s) 2020

\begin{abstract}
Objectives To assess the reliability of judging the spatial relation between the inferior alveolar nerve (IAN) and mandibular third molar (MTM) based on MRI or CT/CBCT images.

Methods Altogether, CT/CBCT and MRI images of 87 MTMs were examined twice by 3 examiners with different degrees of experience. The course of the IAN in relation to the MTM, the presence/absence of a direct contact between IAN and MTM, and the presence of accessory IAN were determined.

Results The IAN was in $>40 \%$ of the cases judged as inferior, while an interradicular position was diagnosed in $<5 \%$ of the cases. The overall agreement was good $(\kappa=0.72)$ and any disagreement between the imaging modalities was primarily among the adjacent regions, i.e., buccal/lingual/interradicular vs. inferior. CT/CBCT judgements presented a very good agreement for the inter- and intrarater comparison $(\kappa>0.80)$, while MRI judgements showed a slightly lower, but good agreement $(\kappa=0.74)$. A direct contact between IAN and MTM was diagnosed in about $65 \%$, but in almost $20 \%$ a disagreement between the judgements based on MRI and CT/CBCT was present resulting in a moderate overall agreement $(\kappa=0.60)$. The agreement between the judgements based on MRI and CT/CBCT appeared independent of the examiner's experience and accessory IAN were described in 10 cases in MRI compared to 3 cases in CT/CBCT images.

Conclusions A good inter- and intrarater agreement has been observed for the assessment of the spatial relation between the IAN and MTM based on MRI images. Further, MRI images might provide advantages in the detection of accessory IAN compared to $\mathrm{CT} / \mathrm{CBCT}$.

Clinical relevance MRI appears as viable alternative to CT/CBCT for preoperative assessment of the IAN in relation to the MTM.
\end{abstract}

Keywords Cone beam computed tomography · Magnetic resonance imaging $\cdot$ Multidetector computed tomography $\cdot$ Oral surgery $\cdot$ Third molar $\cdot$ Mandibular nerve

\section{Introduction}

The inferior alveolar nerve (IAN) is next to the lingual nerve, one of the most endangered anatomical structures during the

Florian Beck

florian.beck@meduniwien.ac.at

1 Division of Oral Surgery, University Clinic of Dentistry, Medical University of Vienna, Vienna, Austria

2 Department of Periodontology, Faculty of Odontology, University of Malmö, Malmö, Sweden surgical removal of mandibular third molars (MTM). Any impairment (i.e., temporary or persistent) of the IAN after MTM removal is reported with an overall incidence of $3.6 \%$; a persistent neurosensory deficit after 6 months is

3 Karl Donath Laboratory for Hard Tissue and Biomaterial Research, Division of Oral Surgery, University Clinic of Dentistry, Medical University of Vienna, Vienna, Austria

4 Department of Biomedical Imaging and Image-guided Therapy, Medical University of Vienna, Vienna, Austria 
observed in $0.9 \%$ [1]. The probability of IAN impairment after MTM removal depends on the spatial relation between the mandibular canal (MC) and the MTM [2] and lack of a bony $\mathrm{MC}$ wall increases the risk of IAN impairment [3]. Hence, preoperative imaging is essential and should include at least a conventional radiograph [4]. In cases with a close relation between the MC and MTM, the exact distance and spatial relation might not be correctly assessed by $2 \mathrm{D}$ images making additional 3D imaging (i.e., computed tomography (CT) or cone beam computed tomography (CBCT)) necessary $[4,5]$. However, CT/CBCT diagnostics relies on the presence of a bony MC wall for locating the IAN. A histomorphometric analysis indicated that the integrity of the MC wall is dependent on the quality/density of the surrounding trabecular bone [6], which explains that in approximately every fifth patient the IAN/MC appears difficult to be identified based on CBCT images [7].

In this context, magnetic resonance imaging (MRI) has been recently proposed as a radiation-free $3 \mathrm{D}$ imaging method for MTM [8]. MRI benefits by directly depicting the neurovascular bundle (NVB) [9] and is therefore independent of the integrity of the $\mathrm{MC}$ wall $[10,11]$. An ex vivo study has proven by superimposition of MRI and CT scans that the geometric accuracy of MRI to display the IAN is comparable to the $\mathrm{CT}$ technique [12]. However, the shape and volume of the NVB were underestimated in a more recent study tracing the IAN in CBCT and MRI [13], and in 7\% of the cases with 1 T MRI, the relation between the MC and impacted MTM was not assessable due to magnetic susceptibility artifacts [14]. Nevertheless, by using 3 T MRI, which has been demonstrated being superior for detection of the courses of brain nerves compared to 1.5 T MRI [15], the accurate visualization of the mandibular branch of the trigeminal nerve has been corroborated $[16,17]$.

The increasing acquisition and use of 3D radiographic imaging within dentistry (e.g., for implant treatment planning [18] and preoperative assessment of MTM [19, 20]) is raising the overall ionizing radiation exposure of the population, i.e., an additional increase of the cancer incidence of 0.46 per year was estimated due to applying CBCT imaging prior to MTM removal [21]. Therefore, MRI could be a reasonable alternative for the planning of various surgical procedures within dentistry [22]. However, despite the increasing number of studies reporting on the possibilities of IAN visualization by MRI, only a few studies $[8,14,23]$ have addressed whether MRI is a reliable diagnostic method to evaluate the course of the IAN in relation to the roots of the MTM. These studies, which have used MRI images alone [14] or MRI with either panoramic radiographs [8] or CBCT [23], have only considered the vertical relation between the MTM and the MC on a panoramic overview. However, from a clinical point of view, the whole $3 \mathrm{D}$ volume including the bucco-lingual relation is important and might affect the surgical approach [24]. Thus, the aim of this study was to assess the reliability of judging the spatial relation between IAN/MC and the roots of the MTM based on orthoradial slices of MRI or CT/CBCT images.

\section{Material and Methods}

\section{Study design and study population}

This cross-sectional study was conducted at the Department of Radiology of the University Clinic of Dentistry, Medical University of Vienna (Austria) between 2017 and 2018. The protocol was approved by the local ethics committee (EK-Nr.: 1487/2017) and all patients provided written informed consent; reporting complies with the STROBE ("Strengthening the Reporting of Observational studies in Epidemiology") guidelines (Appendix 1). Panoramic radiographs of patients consulting the dental clinic for surgical removal of MTM are routinely screened for a close relation between the IAN/MC and the MTM according to the criteria of Rood and Shehab [25] (i.e., darkening of the root, deflected roots, narrowing of the root, dark and bifid root, interruption of the white line(s), diversion of the MC, narrowing of the MC). All patients, which were based on these criteria selected for additional 3D imaging to clarify the spatial relation between the IAN/MC and MTM, were invited to participate, i.e., only patients deemed independent of the present study as in the need of $3 \mathrm{D}$ imaging were considered for inclusion. The following exclusion criteria were defined: (1) < 18 years; (2) installation of any metallic medical device (i.e., aneurysm clip, cochlea implant, defibrillator, insulin/patient-controlled analgesia pump, intrauterine device, intravascular stents/catheter, orthodontic appliance, orthopedic device, valvular transplant); (3) pregnancy; (4) anxiety/restlessness; (5) claustrophobia; (6) tattoos involving metallic particles; and (7) permanent piercings or ear-rings.

\section{Image acquisition-CT/CBCT}

According to the clinic's routine, which are following the "ALARA" (As Low As Reasonably Achievable) principle, either a CBCT or a CT was performed. Specifically, if both MTM were indicated for 3D imaging, a standard dental CT protocol was performed [26] (Somatom Sensation 4, Siemens Healthcare, Erlangen, Germany; $80 \mathrm{mAs}, 120 \mathrm{kV}$, slice thickness: $0.5 \mathrm{~mm}$, FOV: $100-120 \mathrm{~mm}$, table feed: $1 \mathrm{~mm}$, convolution kernel: $\mathrm{U} 70 \mathrm{u}$ ), while in cases of a single MTM, a CBCT scan was recorded (3D Accuitomo XYZ Slice View Tomograph, J. Morita Mfg. Corp., Kyoto, Japan; FOV: 40 $\mathrm{mm}$, slice thickness: $0.25 \mathrm{~mm}$ ). Axial slices from the CT scans were transformed to orthoradial multiplanar reconstructions (MPR) using a semi-automatic line drawn within the center of the jaw by 2 radiologic technologists, who have both $>20$ 
years of experience. The horizontal plane on sagittal MPR was tilted according to Down's mandibular plane (a tangent through the gonial angle and the lowest point of the symphysis).

\section{Image acquisition-MRI}

MRI imaging was performed on a 3-T whole-body MRI scanner (MAGNETOM Skyra, Siemens Healthcare, Erlangen, Germany) using a 64-channel head/neck coil (Head/Neck 64 whole brain DSI, Siemens Healthcare, Erlangen, Germany). Two sequences (axial PD T2 TSE FS, coronal PD TSE FS) were selected by a radiologist (A.G.), specialized in musculoskeletal imaging, from the standard investigation protocol of the jaw region (Table 1, Fig. 1). All sequences were fat saturated to minimize the influence of fatty tissue from cancellous bone on IAN detection [27].

The images were imported in the OsiriX® DICOM image viewer (Pixmeo, SARL, Bernex, Switzerland) for further evaluation. Coronal PD TSE FS scans were transformed and tilted to orthoradial slices by using the "3D MPR" tool by a dental student (S.A.) under close supervision of an experienced radiologist (A.G.). For both imaging modalities, the orthoradial MPR have been produced prior to any assessment, i.e., all examiners judged the same reconstructions.

\section{Image evaluation and assessed parameters}

Image evaluation was performed independently on orthoradial MPR by 3 examiners with a different radiological background: (1) a radiologist (A.G.; > 15 years of experience), (2) an oral surgeon (F.B.; > 7 years of experience), and (3) a

Table 1 MRI sequences and acquisition parameters.

\begin{tabular}{lll}
\hline & Axial PD T2 TSE & Coronal PD TSE \\
\hline FOV & $220 \times 220 \mathrm{~mm}$ & $154 \times 170 \mathrm{~mm}$ \\
Matrix & $320 \times 320$ & $320 \times 232$ \\
Voxel size & $0.34 \times 0.34 \times 2 \mathrm{~mm}^{3}$ & $0.27 \times 0.27 \times 2 \mathrm{~mm}^{3}$ \\
TR & $4060 \mathrm{~ms}$ & $2780 \mathrm{~ms}$ \\
TE & $9.5 \mathrm{~ms}$ & $10 \mathrm{~ms}$ \\
Averages & 1 & 3 \\
Gap & $2.6 \mathrm{~mm}$ & $2.2 \mathrm{~mm}$ \\
Slice Thickness & $2 \mathrm{~mm}$ & $2 \mathrm{~mm}$ \\
Flip angle & $138^{\circ}$ & $141^{\circ}$ \\
Bandwidth & $250 \mathrm{~Hz} / \mathrm{Px}$ & $300 \mathrm{~Hz} / \mathrm{Px}$ \\
Acquisition time & $4: 15(\mathrm{~min}: \mathrm{sec})$ & $6: 29(\mathrm{~min}: \mathrm{sec})$ \\
FS & $\checkmark$ & $\checkmark$ \\
Acquisition type & $2 \mathrm{D}$ & $2 \mathrm{D}$
\end{tabular}

FOV field of view, $F S$ fat saturated, $P D$ proton density, $T E$ echo time, $T R$ repetition time, TSE turbo spin echo dental student (S.A.; last year of education). In a first step, all MRI scans were evaluated. Approximately 14 days later, all $\mathrm{CT} / \mathrm{CBCT}$ scans were assessed, assuring that the evaluation of the MRI and CT/CBCT images of the same patient was performed independently and the raters had no possibility to change any previous judgement, i.e., each rating was immediately finalized and closed. The entire evaluation process was repeated by all 3 examiners after approximately 4 weeks. All evaluations were performed in the same room under standardized conditions on the same screen with the same settings. The following parameters were assessed on the MRI and CT/ CBCT images:

- Course of the IAN/MC (primary outcome parameter): The course of the IAN/MC was classified according to Ghaeminia et al. [28] as (1) lingual, (2) interradicular, (3)

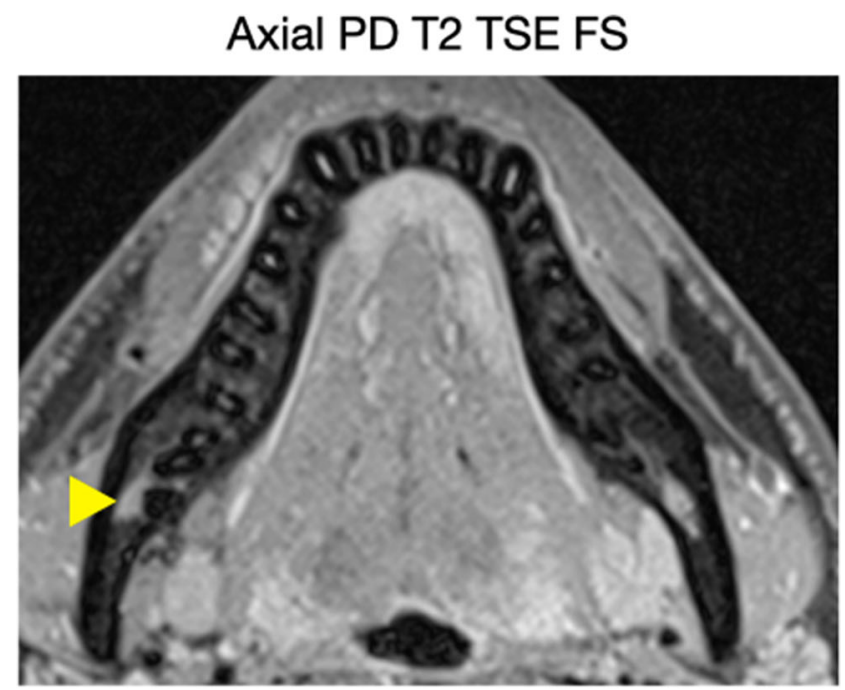

Coronal PD TSE FS

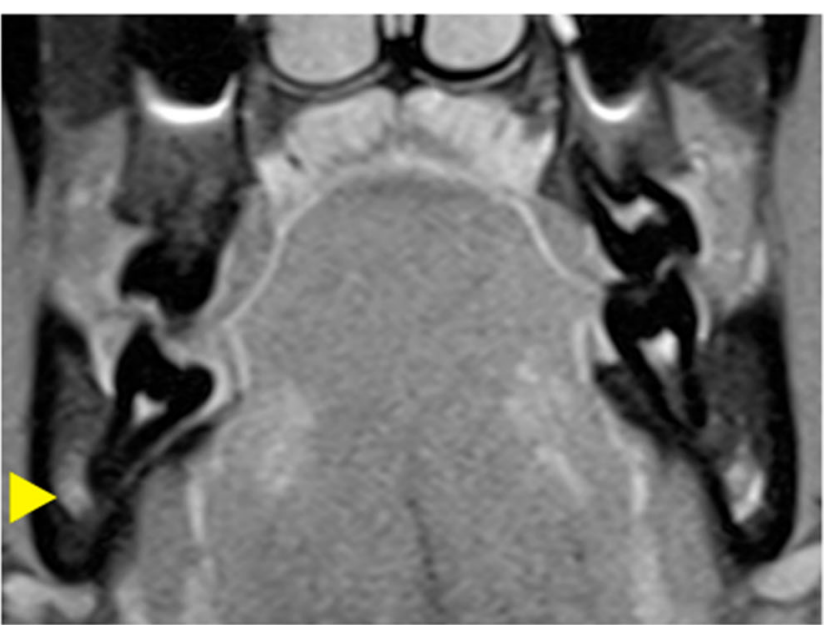

Fig. 1 Two sequences (axial PD T2 TSE FS, coronal PD TSE FS) were selected for the identification of the IAN, which depicted teeth and cortical bone as hypointense, and the IAN (indicated by the yellow arrow), the pulp chamber, the periodontal ligament, small arteries, and dental follicles coronal to the MTM as hyperintense structures 
buccal, or (4) inferior position (Fig. 2). The examiners were instructed to use for the classification the slice representing the closest position between the IAN/MC and the roots of the MTM. Courses matching 2 groups/ classes (e.g., buccal and inferior) were allocated to the aspect, which harbored the greater cross-sectional area of the IAN; if cases were deemed as exactly matching 2 groups/classes (e.g., the cross-section was deemed as $50 \%$ buccal and $50 \%$ inferior), they were allocated to the aspect, which had a higher likelihood of IAN exposure during the surgical procedure.

- Presence/absence of a direct contact between the IAN and the roots of the MTM: Presence of a direct contact between the IAN and the roots of the MTM was defined as the absence of bone tissue between those 2 structures (Fig. 3).

- Presence of any accessory IAN/MC in the region of the MTM (Fig. 4)

In cases with a distal and mesial root, the course of the IAN/MC and the presence/absence of a direct contact were judged for each aspect separately; however, the exact root configuration was not part of the present study. Additionally, the following parameters were recorded: age, gender, and side of the investigated MTM. Prior to any assessment, the 3 examiners performed a calibration session based on 20 randomly chosen $\mathrm{CT} / \mathrm{CBCT}$ and MRI images to discuss and evaluate the above-listed parameters and to dissolve any ambiguity.

\section{Sample size calculation}

A sample size calculation was performed based on the likelihood of classifying the course of the IAN, the number of raters $(n=3)$, and an estimation of $\kappa_{\mathrm{E} 0}=0.6$ from the literature [11, $16,29]$. A lower limit of $\kappa_{\text {lower }}=0.5$ using the formula of Rotondi and Donner [30] was assumed in order to achieve a clinically relevant width with a $95 \%$ confidence interval (CI). The calculated sample size was 68 MTM.

\section{Statistical analysis}

Fleiss' kappa $(\kappa)$ coefficients [31] were calculated for the judgements based on different imaging modalities (i.e., CT/ CBCT vs. MRI) and for inter- and intrarater reliability of the 3 examiners for (1) the course of the IAN/MC and for (2) the presence/absence of a direct contact between the IAN and the roots of the MTM. The $95 \%$ CI of those coefficients were calculated by applying a hierarchical percentile bootstrap on the patient- and rater-level, in order to respect the structure of the data [32]. $P$ values were computed by inverting those CI.
Fig. 2 Classification of the course of the IAN/MC (indicated by the yellow arrow) as buccal, inferior, lingual, or interradicular. b, buccal aspect; 1, lingual aspect
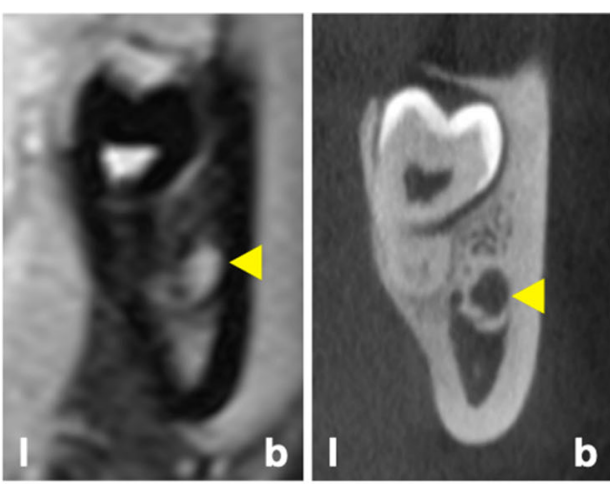

1. buccal course

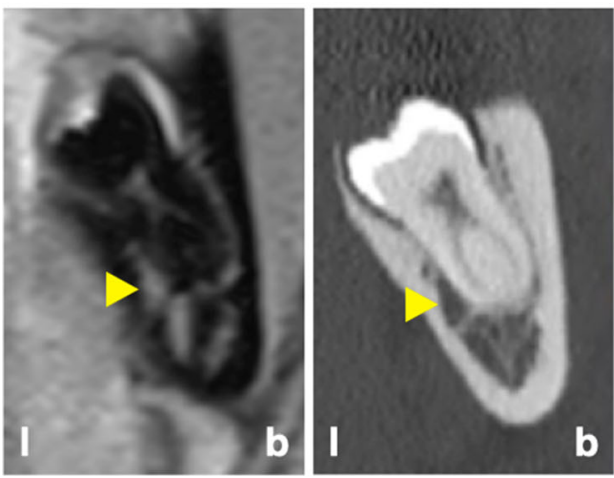

3. lingual course

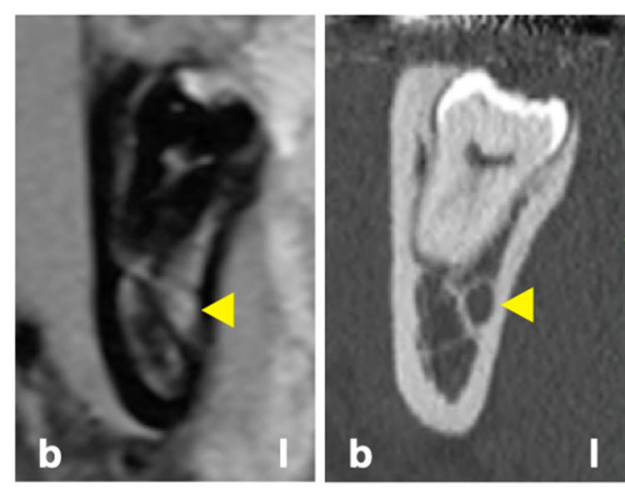

2. inferior course
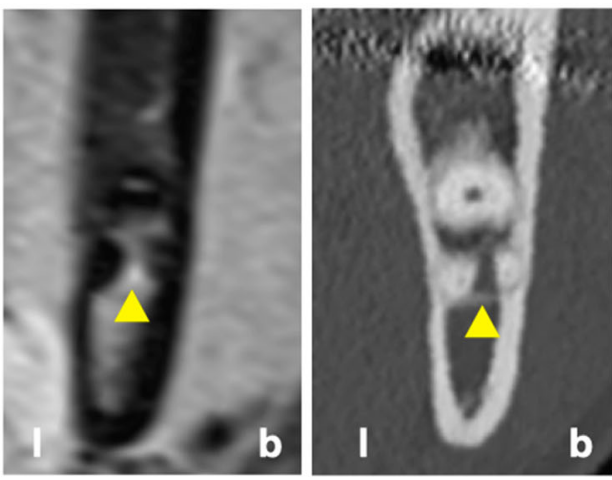

4. interradicular course 
Fig. 3 Presence/absence of bone tissue between the IAN/MC (indicated by the yellow arrow) and the roots of the MTM
MRI
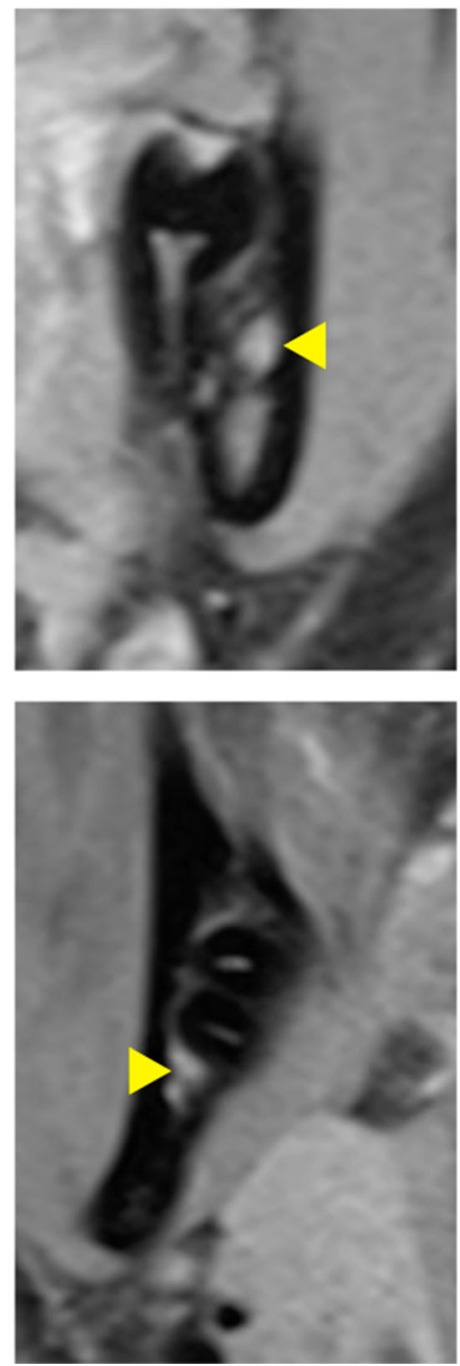

CT/CBCT
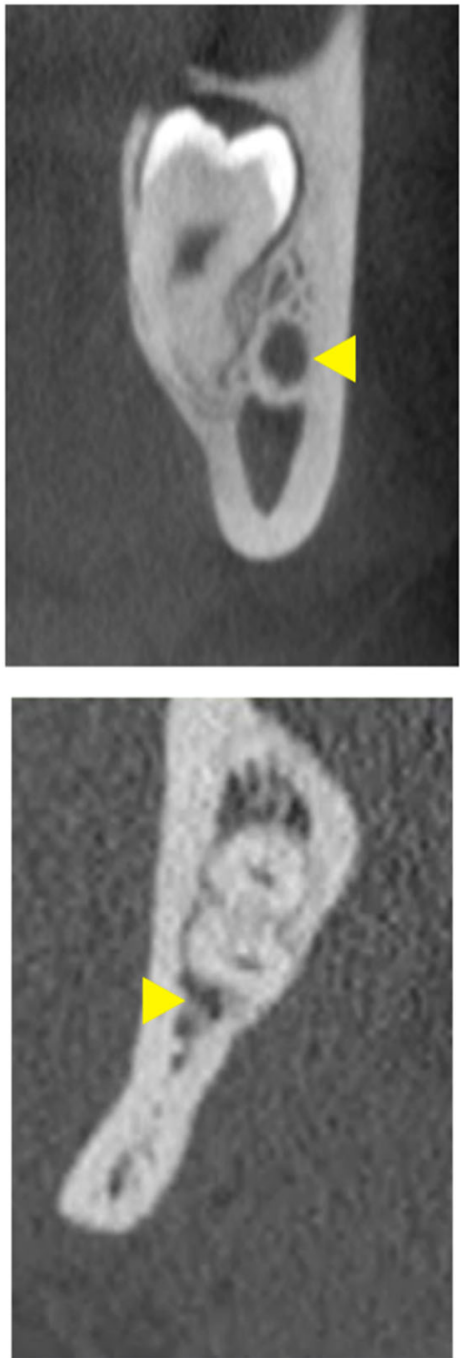

The $\kappa$ values were interpreted as (1) poor $(<0.2)$, (2) fair (0.21-0.4), (3) moderate (0.41-0.6), (4) good (0.61-0.8), and (5) very good (0.81-1.0) [33]. Further, the presence of an accessory IAN/MC in the region of the MTM was descriptively reported. All computations were done using $\mathrm{R}$ version 3.6.1 [34].

\section{Results}

\section{Study population}

The final study population consisted of 53 patients ( 33 female, 20 male; $29.5 \pm 6.5$ years) with 87 MTM, who completed both examinations (i.e., CT/CBCT and MRI) prior to MTM removal. Nineteen participants contributed with CBCT images of a single MTM, while in 34 participants, both MTM were examined by CT; the MTM distribution left to right was 39 to 48.

\section{MRI imaging technique}

The MRI sequences (axial PD T2 TSE FS, coronal PD TSE FS), which were used for the identification of the IAN, depicted teeth and cortical bone as hypointense structures. The IAN, the pulp chamber (including the root canals), the periodontal ligament, small branches of arteries supplying the gingiva and teeth (Rami gingivales inferiores, Rami dentales inferiores), and dental follicles coronal to the MTM were displayed as hyperintense (Fig. 1). In none of the participants, metal artifacts were considered as having an impact on the judgement. 
Fig. 4 A case with the MC lacking a bony wall and with an accessory IAN. Left (CT): the $\mathrm{MC}$ (indicated by the orange arrow) is hardly visible and the accessory IAN (indicated by the yellow arrow) could be missed; right (MRI): both NVB appear clearly hyperintense
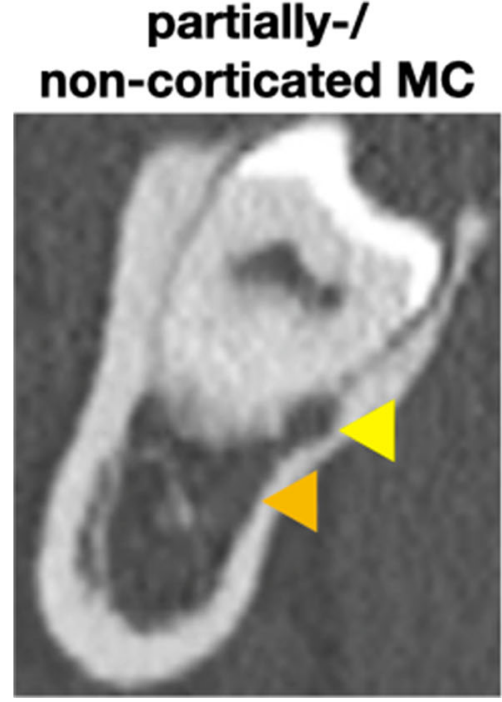

hyperintense IAN and accessory IAN

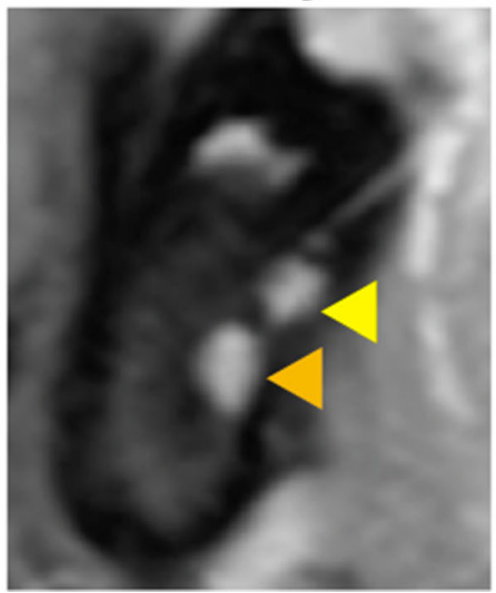

\section{Classification of the course of the IAN/MC}

Table 2 presents the frequency distribution of the course of the IAN/MC in CT/CBCT and MRI based on all available judgements. For both imaging modalities the IAN/MC was most often judged as inferior (CT: $41.8 \%$; MRI: $43.8 \%$ ), followed by the buccal and lingual positions in about 24 to $30 \%$ of the cases, and an interradicular position of the IAN/MC was diagnosed by both imaging modalities in $<5 \%$ of the cases (CT: $3.7 \%$; MRI: $3.1 \%$ ). Further, Table 2 displays that a disagreement between the imaging modalities was primarily among the adjacent regions, i.e., primarily buccal/lingual vs. inferior and in some cases interradicular vs. inferior. Interestingly, in a few cases, the IAN/MC was judged as lingual in the CT/ CBCT images, but as interradicular or buccal in the MRI images. The confusion buccal vs. lingual is based on 2 judgements of the dental student, while all 3 examiners judged the IAN/MC between 1 and 3 times in the MRI images as interradicular, although they judged it as lingual in the $\mathrm{CT} /$ CBCT images.

The overall agreement among all available judgements was $\operatorname{good}(\kappa=0.72)$ with an absolute agreement in $81.5 \%$ of the cases. The CT/CBCT judgements presented in the inter- and intrarater comparison a very good agreement (interrater: $\kappa=$ 0.81 ; intrarater: $\kappa=0.84$ ), while the MRI judgements showed a slightly lower, but good agreement (interrater: $\kappa=0.74$; intrarater: $\kappa=0.74)$. The maximum width of the corresponding $95 \%$ CI was 0.17 (i.e., the difference between $\kappa_{0.975}$ and $\kappa_{0.025}$; Table 3). A comparison of the $\kappa$ values of the 3 examiners when assessing the agreement of their judgements $\mathrm{CT} /$ CBCT vs. MRI proved a good reliability independent of the experience (dental student: $\kappa=0.76$; oral surgeon: $\kappa=0.76$; radiologist: $\kappa=0.80)$ with an absolute agreement ranging from 74.1 to $86.5 \%$.

\section{Direct contact between the IAN/MC and the roots of the MTM}

Table 4 presents the frequency distribution of a direct contact between the IAN/MC and the roots of the MTM in CT/CBCT and MRI based on all available judgements. For both imaging modalities, a direct contact between the IAN/MC and the roots of the MTM was diagnosed in about $65 \%$ (CT: $65 \%$; MRI: $67.2 \%$ ). However, in almost $20 \%$ of the judgements, a disagreement between $\mathrm{CT} / \mathrm{CBCT}$ and MRI was detected.

The overall agreement among all available judgements was moderate $(\kappa=0.60)$ with an absolute agreement in $82 \%$ of the cases. Both image modalities presented a comparable, good inter- and intrarater agreement with the $\kappa$ values ranging from 0.72 to 0.75 . The maximum width of the corresponding $95 \%$ CI was 0.20 (i.e., the difference between $\kappa_{0.975}$ and $\kappa_{0.025}$; Table 5). A comparison of the $\kappa$ values of the 3 examiners when assessing the agreement of their judgements CT/CBCT vs. MRI proved a moderate, close to good reliability independent of the experience (dental student: $\kappa=0.60$; oral surgeon: $\kappa=0.59$; radiologist: $\kappa=0.60$ ) with an absolute agreement ranging from 80.2 to $83.0 \%$.

\section{Presence of an accessory IAN/MC in the region of the MTM}

The presence of an additional, separated hyperintense signal along the course of the IAN (i.e., an accessory IAN) was described in the region of 10 MTM of 10 different patients in the MRI images (Fig. 4). However, the raters did not agree in all cases, i.e., in 5 cases all examiners agreed on the presence of an accessory IAN, while in 2 cases 2 examiners and in 3 cases 1 examiner described an accessory IAN. The judgement of the $\mathrm{CT} / \mathrm{CBCT}$ images revealed only in 3 cases an 
Table 2 Frequency distribution (\%) of the course of the IAN/MC in $\mathrm{CT} / \mathrm{CBCT}$ and MRI based on all available judgements

\begin{tabular}{lllllll}
\hline & & \multicolumn{2}{l}{ Course of the IAN/MC in CT/CBCT } & \multirow{2}{*}{$\begin{array}{l}\text { Total } \\
\text { (MRI) }\end{array}$} \\
\cline { 3 - 6 } & & Buccal & Inferior & Interradicular & Lingual & \\
\hline Course of the IAN/MC in & Buccal & 21.5 & 7.2 & 0 & 0.2 & 28.9 \\
MRI & Inferior & 2.5 & 33.9 & 1.1 & 6.3 & 43.8 \\
& Interradicular & 0 & 0 & 2.6 & 0.5 & 3.1 \\
\multirow{2}{*}{ Total (CT/CBCT) } & lingual & 0 & 0.7 & 0 & 23.5 & 24.2 \\
\hline
\end{tabular}

$C T$ computed tomography, $C B C T$ cone beam computed tomography, IAN inferior alveolar nerve, $M C$ mandibular canal, $M R I$ magnetic resonance imaging accessory MC, which was described in 2 cases by 2 examiners and in 1 case by only one examiner. Hence, in total, in 7 out of 87 cases (i.e., in $8.1 \%$ of the cases), an accessory IAN was assumed in the MRI images which was not reported in the CT/ CBCT images (Fig. 4).

\section{Discussion}

Taking the high frequency of MTM removal, the estimated increase of the cancer incidence due to applying CBCT imaging prior to MTM removal [21], and the questionable benefit to actually reduce the risk for neurosensory disturbance by CBCT compared to panoramic radiographs [35] into account, renders MRI as an interesting radiation-free $3 \mathrm{D}$ imaging method. The present study assessed the reliability of judging the spatial relation between IAN/MC and the roots of the MTM based on orthoradial slices of MRI or CT/CBCT images. A slightly lower inter- and intrarater agreement has been recorded for MRI compared to CT/CBCT images (i.e., $\kappa$ of 0.74 vs. $0.81-0.84$, respectively) when determining the course of the IAN/MC, but the recorded $\kappa$ values still indicate a good reliability for the judgement of MRI images. Additionally, the agreement between the imaging modalities appeared independent of the examiner's experience and MRI might offer advantages in selected cases with an accessory IAN. Herein, in about $8 \%$ of the cases, an accessory IAN was described in

Table 3 Kappa (K) values for the course of the IAN/MC

\begin{tabular}{llllll}
\hline & & $\kappa$ & $\kappa_{0.025}$ & $\kappa_{0.975}$ & $P$ value \\
\hline Overall & & 0.72 & 0.65 & 0.79 & $<0.001$ \\
CT/CBCT & Interrater & 0.81 & 0.76 & 0.86 & $<0.001$ \\
& Intrarater & 0.84 & 0.78 & 0.88 & $<0.001$ \\
MRI & Interrater & 0.74 & 0.67 & 0.80 & $<0.001$ \\
& Intrarater & 0.74 & 0.64 & 0.81 & $<0.001$
\end{tabular}

$C T$ computed tomography, $C B C T$ cone beam computed tomography, $M R I$ magnetic resonance imaging
MRI images, which was not recorded in CT/CBCT images. The judgement of the direct contact between the IAN/MC and the roots of the MTM showed a higher disagreement between the imaging modalities (i.e., in almost $20 \%$ of the cases), which might be due to the fact that $\mathrm{CT} / \mathrm{CBCT}$ and MRI is actually depicting the MC or the IAN, respectively.

In this context, $\mathrm{CT} / \mathrm{CBCT}$ relies on the presence of a MC wall for locating the IAN, i.e., in cases with a minimal amount of bone surrounding the IAN, it can be difficult to identify the $\mathrm{MC}$ in $\mathrm{CBCT}$ images [7]. In contrast, MRI displays directly the NVB including the IAN. To date, there is no standard term regarding what is actually visualized by MRI. Nasel et al. [9] have previously stated that the neural and vascular structures within the MC could not be distinguished and thus referred to the term "NVB." Since then, "NVB" [36-39], "IAN" [8, 17, 40, 41], "mandibular nerve" [10, 12], or "MC" [11, 14, 23, 29] were used synonymously in various publications. The crosssectional area of the NVB in the region of the MTM measured by histomorphometry is about $13.45 \pm 2.23 \mathrm{~mm}^{2}$; thereof, the IAN and the inferior alveolar artery (IAA) represent 32.4 and $4.5 \%$ of the area, respectively [39]. The IAN itself is composed of a larger mental $(2 / 3)$ and a smaller incisive $(1 / 3)$ branch [42]. In regard to the structural dimensions within the NVB (i.e., with the IAN being 6 to 7 times larger than the IAA), it is reasonable to assume that the hyperintense signal in MRI is mostly expressed by the IAN. However, the NVB as

Table 4 Frequency distribution (\%) of a direct contact between the IAN/MC and the roots of the MTM in CT/CBCT and MRI based on all available judgements

\begin{tabular}{lllll}
\hline & & \multicolumn{2}{l}{ Contact CT/CBCT } & \multirow{2}{*}{ Total (MRI) } \\
\cline { 2 - 3 } & & Yes & No & \\
\hline Contact MRI & Yes & 57.1 & 10.1 & 67.2 \\
& No & 7.9 & 24.9 & 32.8 \\
Total (CT/CBCT) & 65 & 35 & 100 \\
\hline
\end{tabular}

$C T$ computed tomography, $C B C T$ cone beam computed tomography, $M R I$ magnetic resonance imaging 
Table 5 Kappa $(\kappa)$ values of a direct contact between the IAN/MC and the roots of the MTM

\begin{tabular}{lllll}
\hline & & $\kappa$ & $\kappa_{0.025}$ & $\kappa_{0.975}$ \\
\hline Overall & & 0.60 & 0.49 & 0.69 \\
CT/CBCT & Interrater & 0.75 & 0.68 & 0.81 \\
& Intrarater & 0.72 & 0.63 & 0.79 \\
MRI & Interrater & 0.72 & 0.63 & 0.81 \\
& Intrarater & 0.74 & 0.65 & 0.83 \\
\hline
\end{tabular}

$C T$ computed tomography, $C B C T$ cone beam computed tomography, $M R I$ magnetic resonance imaging

total does not necessarily fill the whole volume of the MC (Fig. 5). Additionally, the structures within the NVB might change their spatial relation to each other as it has been shown for the IAA, i.e., the IAA changes its position within the MC on average about 4 times being at the MTM most often in a cranial position [43]. Altogether, this might explain at least partly the higher disagreement observed herein between the imaging modalities for the judgement of a direct contact between the IAN/MC and the roots of the MTM. For example, the IAN might be located within the MC more distant to the roots and not displaying a direct contact in the MRI images, although no bone tissue is displayed between the $\mathrm{MC}$ and the roots in $\mathrm{CT} / \mathrm{CBCT}$ images. Alternatively, the IAN might be located close to the roots and the hyperintense signal interferes with the judgement of the presence/absence of hypointense bone tissue between the IAN and the roots in MRI images. Finally, the superior visualization of hard tissue in CT/CBCT images (e.g., the border between cancellous bone and the root surface) might also play a role.

Any disagreement between the imaging modalities for the course of the IAN/MC was primarily among adjacent regions, i.e., primarily buccal/lingual vs. inferior and in some cases interradicular vs. inferior (Table 2). Specifically, the
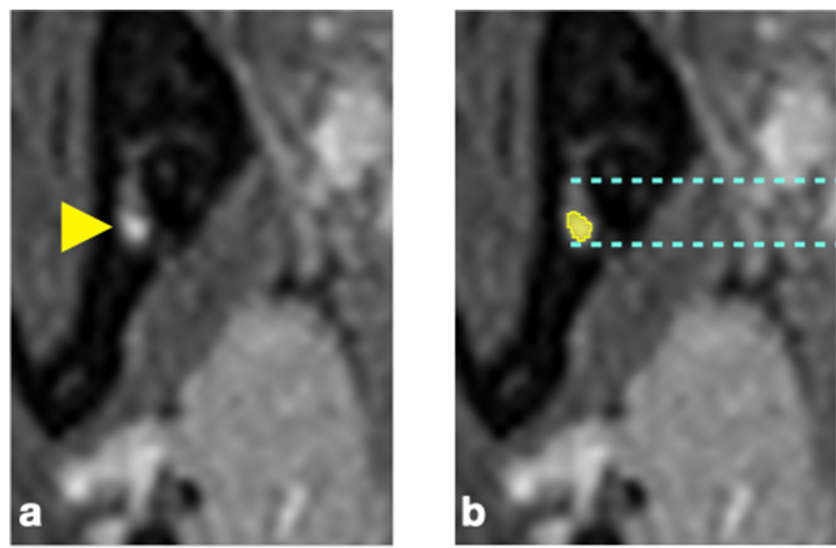

Fig. 5 A case with the NVB (indicated in (a) by the yellow arrow and in (b, c) by the yellow area) not filling the whole volume of the MC (indicated in (c) by the orange dotted line and in (d) by the orange disagreement can be explained for the most part by the "borderline" cases with the IAN/MC matching 2 groups/classes (e.g., lingual and inferior). It was defined herein to allocate the IAN/MC to the aspect, which harbored the greater crosssectional area; however, this implies naturally a certain risk for inter- and intrarater disagreement. Further, the fact that either the MC or the IAN are displayed in CT/CBCT or MRI images, respectively, probably contributes to a potential disagreement among adjacent groups/classes, i.e., as discussed above, that the IAN does not necessarily fill the whole volume of the MC and therefore might be depicted in a slightly different position in MRI images compared to CT/CBCT images. Nevertheless, only in very few cases (i.e., in $0.7 \%$ of all judgements) that the IAN/MC was clearly "misjudged" between the imaging modalities (i.e., a confusion between buccal/lingual, buccal/ interradicular, or lingual/interradicular), which underlines MRI as viable alternative to CT/CBCT. Additionally, the confusion buccal vs. lingual appeared only twice by the examiner with the least experience. Finally, CT/CBCT was considered herein as a "gold standard" as it is the main 3D imaging technique being used if a close relation is suspected on panoramic radiographs [4]. However, one should keep in mind that the "misjudgment" might actually not necessarily occur when judging the MRI images. In fact, MRI is the most commonly used technique to directly visualize the IAN [22] and to precisely localize the IAN including pathologic processes $[9,44-46]$.

To the best of our knowledge, a direct comparison to previous studies is not possible, as this is the first comparison of MRI and CT/CBCT images for a description of the relation of IAN/MC to MTM based on a 3D volume data set. In general, this relation is ideally expressed by both the course of the IAN in relation to the roots of the MTM (i.e., buccal, lingual, inferior, interradicular) and the presence/absence of bone tissue between the IAN and the roots of the MTM [28]. For example, a previous study [8] compared amongst other parameters the vertical position of the MTM in relation to the IAN based on
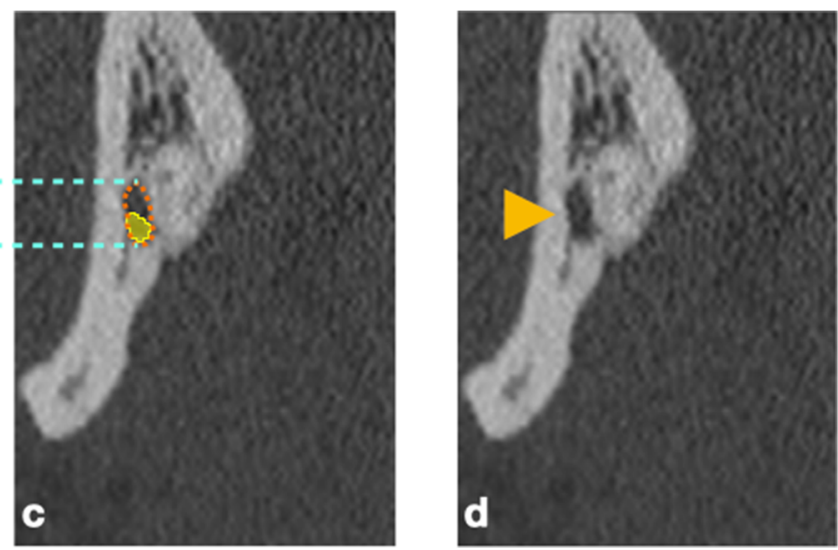

arrow); the corresponding vertical dimension of the MC between both imaging modalities $(\mathbf{b}, \mathbf{c})$ is indicated by the turquoise dotted line 
MRI and panoramic radiographs. They reached an agreement in only $56 \%$ of the cases and the interrater agreement was poor $\left(\kappa_{\mathrm{w}}=0.142\right)$. However, from a clinical point of view, MRI will not replace the panoramic radiograph as primary diagnostic method, but - as shown herein-MRI could replace CT/ $\mathrm{CBCT}$ in cases, where the panoramic radiograph indicated the need for an additional 3D imaging technique to display in detail the vertical and bucco-lingual relation between the IAN/MC and the roots of the MTM without additional ionizing radiation exposure.

Altogether, MRI presents several advantages compared to $\mathrm{CT} / \mathrm{CBCT}$. Besides the obvious ones, such as radiation-free recording and the independency of the presence of a bony $\mathrm{MC}$ wall, MRI might be advantageous in displaying an accessory IAN and/or the lingual nerve. Herein, in 7 out of 87 cases, an accessory IAN was described in the MRI images, which was not visible in the CT/CBCT images (Fig. 4). However, based on the present data, one cannot exclude the possibility of false positive results in the judgement of MRI images for an accessory IAN. Future studies including histological examinations would be required to determine the truth regarding the presence/absence of accessory IAN. In general, the actual lack of a "gold standard" should be considered when interpreting the results herein, i.e., one does actually not know whether the judgement based on MRI or CT/CBCT images is the truth. Further, a recent study explored the feasibility of MRI images to detect and follow the lingual nerve [47]. Visualization of the lingual nerve was not part of the present study and the best sequence to depict the lingual nerve requires future studies. In general, visualization of the lingual nerve prior to MTM removal sounds tempting, as a temporary neurosensory disturbance (up to 13 weeks) is reported in $2 \%$ of the cases [48] and the lingual nerve is not visible in CT/CBCT images. However, one should also keep in mind the limitations of MRI with the most important ones being the general accessibility and the fact that it is a more expensive and time-consuming examination. Specifically, the cost-effectiveness might be difficult to prove, especially as even CT/CBCT still lacks the proof of a benefit to actually reduce the risk for neurosensory disturbance compared to panoramic radiographs only [35].

When evaluating the results of the present study, several limitations should be taken into consideration. First, for both imaging modalities, the orthoradial MPR have been produced prior to any assessment by different persons. Specifically, 2 experienced radiologic technologists were responsible for the CT/CBCT scans and the dental student together with the radiologist for the MRI scans. It can be discussed whether orthoradial MPR performed by different persons may affect the reliability; however, as all examiners judged the same reconstructions, any effect should be minor. In this context, if the examiners would have prepared the MPR themselves, it might have lowered the reliability for both MRI and CT/CBCT. However, this might primarily affect the "borderline" cases with the IAN/MC matching 2 groups/classes. Second, the inclusion of a dental student could be considered as a weakness, yet it allowed us to get an idea about the effect of the examiner's experience. Specifically, it was interesting to see that the agreement between the judgements of both imaging modalities appeared independent of the examiner's experience for the outcome parameters (i.e., course of the IAN/MC and direct contact between the IAN/MC and the MTM). Hence, at least for this specific topic, already a short calibration session prior to committing this study enabled a dental student to read the MRI and $\mathrm{CT} / \mathrm{CBCT}$ images with an agreement comparable to the 2 other examiners (i.e., an oral surgeon and a radiologist). This in turn allows a careful conclusion that one can probably expect a high learning curve when starting to incorporate MRI as an alternative to $\mathrm{CT} / \mathrm{CBCT}$ for assessing the relation between IAN and MTM in daily practice. Third, 68 MTM were assessed herein by CT and only 19 cases by CBCT. As CBCT provides a slightly higher resolution compared to CT [49, 50], an assessment based on CBCT images only might increase the reliability of the "standard" method. Finally, the present study did neither aim to develop new sequences for head and neck MRI nor to compare different sequences. Instead, the investigation was based on sequences of the standard protocol, which are usually available in radiological institutions. Hence, it would be an interesting topic of future studies whether the depiction of the IAN could be further improved, which in turn might improve the reliability of judgements based on MRI images.

In conclusion, the present study demonstrated the suitability of MRI with fat-saturated axial PD T2 TSE and coronal PD TSE sequences for the assessment of the IAN course in relation to the roots of the MTM prior to surgery as a radiological method free of ionizing radiation. Specifically, although the judgement of MRI images showed a slightly lower inter- and intrarater agreement in terms of determining the course of the IAN/MC compared to a judgement based on CT/CBCT images, the reliability can be considered as good. Additionally, the agreement appeared overall independent of the examiner's experience and MRI might have advantages in seldom cases with a second, accessory IAN.

Supplementary Information The online version contains supplementary material available at https://doi.org/10.1007/s00784-020-03716-4.

Acknowledgments The authors express special thankfulness to Anita Masopust, Teresa Keindl, and Helge Schöchtner for their support in performing the MRI and the CT/CBCT scans.

Funding Open Access funding provided by Medical University of Vienna.

\section{Compliance with ethical standards}

Conflict of interest The authors declare that they have no conflict of interest. 
Ethical approval An Institutional Review Board approval for this study (EK-Nr.: 1487/2017) was obtained.

Informed consent Written informed consent was obtained from all patients in this study.

Open Access This article is licensed under a Creative Commons Attribution 4.0 International License, which permits use, sharing, adaptation, distribution and reproduction in any medium or format, as long as you give appropriate credit to the original author(s) and the source, provide a link to the Creative Commons licence, and indicate if changes were made. The images or other third party material in this article are included in the article's Creative Commons licence, unless indicated otherwise in a credit line to the material. If material is not included in the article's Creative Commons licence and your intended use is not permitted by statutory regulation or exceeds the permitted use, you will need to obtain permission directly from the copyright holder. To view a copy of this licence, visit http://creativecommons.org/licenses/by/4.0/.

\section{References}

1. Gülicher D, Gerlach KL (2001) Sensory impairment of the lingual and inferior alveolar nerves following removal of impacted mandibular third molars. Int J Oral Maxillofac Surg 30:306-312. https:// doi.org/10.1054/ijom.2001.0057

2. Palma-Carrio C, Garcia-Mira B, Larrazabal-Moron C, PenarrochaDiago M (2010) Radiographic signs associated with inferior alveolar nerve damage following lower third molar extraction. Med Oral Patol Oral Cirugia Bucal:e886-e890. https://doi.org/10.4317/ medoral.15.e886

3. Park W, Choi J-W, Kim J-Y et al (2010) Cortical integrity of the inferior alveolar canal as a predictor of paresthesia after third-molar extraction. J Am Dent Assoc 141:271-278. https://doi.org/10. 14219/jada.archive.2010.0160

4. Flygare L, Öhman A (2008) Preoperative imaging procedures for lower wisdom teeth removal. Clin Oral Investig 12:291-302. https://doi.org/10.1007/s00784-008-0200-1

5. Ohman A, Kivijärvi K, Blombäck U, Flygare L (2006) Preoperative radiographic evaluation of lower third molars with computed tomography. Dento Maxillo Facial Radiol 35:30-35. https:// doi.org/10.1259/dmfr/58068337

6. Bertl K, Heimel P, Reich KM, Schwarze UY, Ulm C (2014) A histomorphometric analysis of the nature of the mandibular canal in the anterior molar region. Clin Oral Investig 18:41-47. https:// doi.org/10.1007/s00784-013-0961-z

7. Oliveira-Santos C, Capelozza ALÁ, Dezzoti MSG, Fischer CM, Poleti ML, Rubira-Bullen IRF (2011) Visibility of the mandibular canal on CBCT crosssectional images. J Appl Oral Sci 19:240-243. https://doi.org/10.1590/S1678-77572011000300011

8. Kirnbauer B, Jakse N, Rugani P, Schwaiger M, Magyar M (2018) Assessment of impacted and partially impacted lower third molars with panoramic radiography compared to MRI - a proof of principle study. Dentomaxillofacial Radiol 47:20170371. https://doi.org/ 10.1259/dmfr.20170371

9. Nasel C, Gahleitner A, Breitenseher M et al (1998) Localization of the mandibular neurovascular bundle using dental magnetic resonance imaging. Dentomaxillofac Radiol 27:305-307. https://oi. org $/ 10.1038 / \mathrm{sj} / \mathrm{dmfr} / 4600379$

10. Chau A (2012) Comparison between the use of magnetic resonance imaging and conebeam computed tomography for mandibular nerve identification. Clin Oral Implants Res 23:253-256. https:// doi.org/10.1111/j.1600-0501.2011.02188.x
11. Imamura H, Sato H, Matsuura T, Ishikawa M, Zeze R (2004) A comparative study of computed tomography and magnetic resonance imaging for the detection of mandibular canals and crosssectional areas in diagnosis prior to dental implant treatment. Clin Implant Dent Relat Res 6:75-81. https://doi.org/10.1111/j.17088208.2004.tb00029.x

12. Eggers G, Rieker M, Fiebach J, Kress B, Dickhaus H, Hassfeld S (2005) Geometric accuracy of magnetic resonance imaging of the mandibular nerve. Dentomaxillofacial Radiol 34:285-291. https:// doi.org/10.1259/dmfr/89236515

13. Agbaje JO, Casteele EVD, Salem AS, Anumendem D (2016) Tracking of the inferior alveolar nerve : its implication in surgical planning. Clin Oral Investig 10:2213-2220. https://doi.org/10. 1007/s00784-016-2014-x

14. Ferretti F, Malventi M, Malasoma R (2009) Dental magnetic resonance imaging: study of impacted mandibular third molars. Dentomaxillofacial Radiol 38:387-392. https://oi.org/10.1259/ dmfr/29929241

15. Fischbach F, Müller M, Bruhn H (2008) Magnetic resonance imaging of the cranial nerves in the posterior fossa: a comparative study of T2-weighted spin-echo sequences at 1.5 and 3.0 Tesla. Acta Radiol 49:358-363. https://doi.org/10.1080/ 02841850701824127

16. Assaf AT, Zrnc TA, Remus CC, Schönfeld M, Habermann CR, Riecke B, Friedrich RE, Fiehler J, Heiland M, Sedlacik J (2014) Evaluation of four different optimized magnetic-resonance-imaging sequences for visualization of dental and maxillo-mandibular structures at 3 T. J Cranio-Maxillofac Surg 42:1356-1363. https://doi. org/10.1016/j.jcms.2014.03.026

17. Cassetta M, Pranno N, Pompa V, Barchetti F, Pompa G (2014) High resolution 3-T MR imaging in the evaluation of the trigeminal nerve course. Eur Rev Med Pharmacol Sci 18:257-264

18. Bornstein MM, Brügger OE, Janner SFM et al (2015) Indications and frequency for the use of cone beam computed tomography for implant treatment planning in a specialty clinic. Int J Oral Maxillofac Implants 30:1076-1083. https://oi.org/10.11607/jomi. 4081

19. Sivolella S, Boccuzzo G, Gasparini E, de Conti G, Berengo M (2012) Assessing the need for computed tomography for lowerthird-molar extraction: a survey among 322 dentists. Radiol Med (Torino) 117:112-124. https://doi.org/10.1007/s11547-011-0678-5

20. Korkmaz YT, Kayıpmaz S, Senel FC, Atasoy KT, Gumrukcu Z (2017) Does additional cone beam computed tomography decrease the risk of inferior alveolar nerve injury in high-risk cases undergoing third molar surgery? Does CBCT decrease the risk of IAN injury? Int J Oral Maxillofac Surg 46:628-635. https://doi.org/10. 1016/j.ijom.2017.01.001

21. Petersen LB, Olsen KR, Matzen LH, Vaeth M, Wenzel A (2015) Economic and health implications of routine CBCT examination before surgical removal of the mandibular third molar in the Danish population. Dentomaxillofacial Radiol 44:20140406. https://doi. org/10.1259/dmfr.20140406

22. Weckx A, Agbaje JO, Sun Y, Jacobs R, Politis C (2016) Visualization techniques of the inferior alveolar nerve (IAN): a narrative review. Surg Radiol Anat 38:55-63. https://doi.org/10. 1007/s00276-015-1510-Z

23. Kang Y, Hua C, Wu B, Zhou Z, Xu Y, Wu P, Wang H, Hong N (2018) Investigation of zero TE MR in preoperative planning in dentistry. Magn Reson Imaging 54:77-83. https://doi.org/10. 1016/j.mri.2018.07.007

24. Ghaeminia H, Meijer GJ, Soehardi A, Borstlap WA, Mulder J, Vlijmen OJC, Bergé SJ, Maal TJJ (2011) The use of cone beam CT for the removal of wisdom teeth changes the surgical approach compared with panoramic radiography: a pilot study. Int J Oral Maxillofac Surg 40:834-839. https://doi.org/10.1016/j.ijom.2011. 02.032 
25. Rood JP, Shehab BA (1990) The radiological prediction of inferior alveolar nerve injury during third molar surgery. Br J Oral Maxillofac Surg 28:20-25. https://doi.org/10.1016/0266-4356(90) 90005-6

26. Gahleitner A, Podesser B, Schick S, Watzek G, Imhof H (2004) Dental CT and orthodontic implants: imaging technique and assessment of available bone volume in the hard palate. Eur J Radiol 51: 257-262. https://doi.org/10.1016/j.ejrad.2003.11.021

27. Gray CF, Redpath TW, Smith FW, Staff RT (2003) Advanced imaging: magnetic resonance imaging in implant dentistry. Clin Oral Implants Res 14:18-27. https://doi.org/10.1034/j.1600-0501. 2003.140103.x

28. Ghaeminia H, Meijer GJ, Soehardi A, Borstlap WA, Mulder J, Bergé SJ (2009) Position of the impacted third molar in relation to the mandibular canal. Diagnostic accuracy of cone beam computed tomography compared with panoramic radiography. Int J Oral Maxillofac Surg 38:964-971. https://doi.org/10.1016/j.ijom. 2009.06.007

29. Manoliu A, Ho M, Nanz D, Dappa E, Boss A, Grodzki DM, Liu W, Chhabra A, Andreisek G, Kuhn FP (2016) MR neurographic orthopantomogram: Ultrashort echo-time imaging of mandibular bone and teeth complemented with high-resolution morphological and functional MR neurography: MR neurographic orthopantomogram. J Magn Reson Imaging 44:393-400. https:// doi.org/10.1002/jmri.25178

30. Rotondi MA, Donner A (2012) A confidence interval approach to sample size estimation for interobserver agreement studies with multiple raters and outcomes. J Clin Epidemiol 65:778-784. https://doi.org/10.1016/j.jclinepi.2011.10.019

31. Shrout PE, Fleiss JL (1979) Intraclass correlations: uses in assessing rater reliability. Psychol Bull 86:420-428. https://doi.org/10.1037// 0033-2909.86.2.420

32. Davison AC, Hinkley DV (1997) Bootstrap methods and their application. Cambridge University Press, Cambridge

33. Altman DG (1990) Practical statistics for medical research. CRC press

34. R Core Team (2019) R: a language and environment for statistical computing. (Version 3.6.1). R Foundation for Statistical Computing, Vienna

35. de Toledo Telles-Araújo G, Peralta-Mamani M, Caminha RDG et al (2020) CBCT does not reduce neurosensory disturbances after third molar removal compared to panoramic radiography: a systematic review and meta-analysis. Clin Oral Investig 24:1137-1149. https://doi.org/10.1007/s00784-020-03231-6

36. Kress B, Gottschalk A, Anders L, Stippich C, Palm F, Bähren W, Sartor K (2004) High-resolution dental magnetic resonance imaging of inferior alveolar nerve responses to the extraction of third molars. Eur Radiol 14:1416-1420. https://doi.org/10.1007/s00330004-2285-5

37. Wamasing P, Deepho C, Watanabe H, Hayashi Y, Sakamoto J, Kurabayashi $T$ (2018) Imaging the bifid mandibular canal using high resolution MRI. Dentomaxillofacial Radiol 48:20180305. https://doi.org/10.1259/dmfr.20180305

38. Tantanapornkul W, Okouchi K, Fujiwara Y, Yamashiro M, Maruoka Y, Ohbayashi N, Kurabayashi T (2007) A comparative study of cone-beam computed tomography and conventional panoramic radiography in assessing the topographic relationship between the mandibular canal and impacted third molars. Oral Surg Oral Med Oral Pathol Oral Radiol Endodontol 103:253-259. https://doi.org/10.1016/j.tripleo.2006.06.060
39. Yu SK, Lee MH, Jeon YH, Chung YY, Kim HJ (2016) Anatomical configuration of the inferior alveolar neurovascular bundle: a histomorphometric analysis. Surg Radiol Anat 38:195-201. https://doi.org/10.1007/s00276-015-1540-6

40. Krasny A, Krasny N, Prescher A (2012) Anatomic variations of neural canal structures of the mandible observed by 3-Tesla magnetic resonance imaging. J Comput Assist Tomogr 36:150-153. https://doi.org/10.1097/RCT.0b013e3182436c6d

41. Probst M, Richter V, Weitz J, Kirschke JS, Ganter C, Troeltzsch M, Nittka M, Cornelius CP, Zimmer C, Probst FA (2017) Magnetic resonance imaging of the inferior alveolar nerve with special regard to metal artifact reduction. J Cranio-Maxillofac Surg 45:558-569. https://doi.org/10.1016/j.jcms.2017.01.009

42. Hur M, Kim H, Won S et al (2011) Topography and spatial fascicular arrangement of the human inferior alveolar nerve:88-95. https://doi.org/10.1111/j.1708-8208.2011.00335.x

43. Bertl K, Hirtler L, Dobsak T, Heimel P, Gahleitner A, Ulm C, Plenk $\mathrm{H} \mathrm{Jr}$ (2015) Radiological assessment of the inferior alveolar artery course in human corpse mandibles. Eur Radiol 25:1148-1153. https://doi.org/10.1007/s00330-014-3484-3

44. Kreutner J, Hopfgartner A, Weber D, Boldt J, Rottner K, Richter E, Jakob PM, Haddad D (2016) High isotropic resolution magnetic resonance imaging of the mandibular canal at $1.5 \mathrm{~T}$ : a comparison of gradient and spin echo sequences. Dentomaxillofacial Radiol 46: 20160268. https://doi.org/10.1259/dmfr.20160268

45. Kress B, Nissen S, Gottschalk A, Anders L, Wentzler C, Solbach T, Palm F, Bähren W, Sartor K (2003) High-resolution MR technique allowing visualization of the course of the inferior alveolar nerve along cystic processes. Eur Radiol 13:1612-1614. https://doi.org/ 10.1007/s00330-003-1837-4

46. Krasny A, Krasny N, Prescher A (2011) Study of inferior dental canal and its contents using high-resolution magnetic resonance imaging. Surg Radiol Anat 34:687-693. https://doi.org/10.1007/ s00276-011-0910-y

47. Burian E, Probst FA, Weidlich D, Cornelius CP, Maier L, Robl T, Zimmer C, Karampinos DC, Ritschl LM, Probst M (2019) MRI of the inferior alveolar nerve and lingual nerve - anatomical variation and morphometric benchmark values of nerve diameters in healthy subjects. Clin Oral Investig. 24:2625-2634. https://doi.org/10. 1007/s00784-019-03120-7

48. Valmaseda-Castellón E, Berini-Aytés L, Gay-Escoda C (2000) Lingual nerve damage after third lower molar surgical extraction. Oral Surg Oral Med Oral Pathol Oral Radiol Endodontol 90:567573. https://doi.org/10.1067/moe.2000.110034

49. Veldhoen S, Schöllchen M, Hanken H, Precht C, Henes FO, Schön G, Nagel HD, Schumacher U, Heiland M, Adam G, Regier M (2017) Performance of cone-beam computed tomography and multidetector computed tomography in diagnostic imaging of the midface: a comparative study on phantom and cadaver head scans. Eur Radiol 27:790-800. https://doi.org/10.1007/s00330-016-43872

50. Minami S, Ohnishi T, Sano T, Sugiura K, Nakayama E (2015) Comparison between cone-beam CT and multidetector-row CT by ROC analysis regarding diagnostic accuracy for artificial alveolar bone defects in the mandibular molar region. Oral Radiol 31:97104. https://doi.org/10.1007/s11282-014-0189-x

Publisher's note Springer Nature remains neutral with regard to jurisdictional claims in published maps and institutional affiliations. 